PROCEEDINGS OF THE

AMERICAN MATHEMATICAL SOCIETY

Volume 137, Number 10, October 2009, Pages 3335-3342

S 0002-9939(09)09828-1

Article electronically published on May 13, 2009

\title{
ON ISOMORPHIC CLASSIFICATIONS OF SPACES OF COMPACT OPERATORS
}

\author{
ELÓI MEDINA GALEGO
}

(Communicated by Nigel J. Kalton)

\begin{abstract}
We prove an extension of the classical isomorphic classification of Banach spaces of continuous functions on ordinals. As a consequence, we give complete isomorphic classifications of some Banach spaces $\mathcal{K}\left(X, Y^{\eta}\right), \eta \geq \omega$, of compact operators from $X$ to $Y^{\eta}$, the space of all continuous $Y$-valued functions defined in the interval of ordinals $[1, \eta]$ and equipped with the supremum norm. In particular, under the Continuum Hypothesis, we extend a recent result of C. Samuel by classifying, up to isomorphism, the spaces $\mathcal{K}\left(X^{\xi}, c_{0}(\Gamma)^{\eta}\right)$, where $\omega \leq \xi<\omega_{1}, \eta \geq \omega, \Gamma$ is a countable set, $X$ contains no complemented copy of $l_{1}, X^{*}$ has the Mazur property and the density character of $X^{* *}$ is less than or equal to $\aleph_{1}$.
\end{abstract}

\section{Introduction And Statement of the main RESUlt}

Let $X$ be a Banach space and $K$ a compact Hausdorff space. By $C(K, X)$ we denote the Banach space of all continuous $X$-valued functions defined on $K$ and equipped with the supremum norm. This space will be denoted by $C(K)$ in the case $X=\mathbb{R}$. As in [1, when $K$ is the interval of ordinals $[1, \eta]$ endowed with the order topology, this space will be indicated by $X^{\eta}$. Given Banach spaces $X$ and $Y$, $\mathcal{K}(X, Y)$ denotes the Banach space of compact operators from $X$ to $Y$.

For a fixed a set $\Gamma$ and a Banach space $X, c_{0}(\Gamma, X)$ denotes the Banach space of all $X$-valued functions $f$ defined on $\Gamma$ such that for all $\epsilon>0$ there exists a finite subset $F_{\epsilon}$ of $\Gamma$ with $\|f(\gamma)\|<\epsilon$ for all $\gamma \notin F_{\epsilon}$, under the supremum norm, and $l_{p}(\Gamma, X), 1 \leq p<\infty$, denotes the subset of $c_{0}(\Gamma, X)$ consisting of those $f$ for which $\sum_{\gamma \in \Gamma}\|f(\gamma)\|^{p}<\infty$, under the norm $\|f\|=\left(\sum_{\gamma \in \Gamma}\|f(\gamma)\|^{p}\right)^{1 / p}$. As usual we also write $c_{0}(\Gamma)$ and $l_{p}(\Gamma)$ for the case $X=\mathbb{R}$, and simply $c_{0}$ and $l_{p}$ when $\Gamma$ is countable infinite.

In a recent paper, C. Samuel [17] provides a complete isomorphic classification of spaces $\mathcal{K}(C(Q), C(Q))$, where $Q$ is a countable compact metric space. One of the main tools used in the proof of this result is the classical theorem of Mazurkiewicz and Sierpiński which states that every countable compact metric space is homeomorphic to an interval of ordinals $[1, \eta]$ with $\omega \leq \eta<\omega_{1}$. Indeed, in [17] it was given a complete isomorphic classification of spaces $\mathcal{K}\left(\mathbb{R}^{\eta}, \mathbb{R}^{\eta}\right)$ with $\omega \leq \eta<\omega_{1}$.

Received by the editors August 28, 2008.

2000 Mathematics Subject Classification. Primary 46B03, 46B25; Secondary 47B10.

Key words and phrases. Isomorphic classifications of spaces of continuous functions, compact operators.

(C)2009 American Mathematical Society Reverts to public domain 28 years from publication 
The present paper is intended to be a continuation of [17. Here we deal with isomorphic classifications of spaces of compact operators in more general settings. More precisely, we are especially concerned with the isomorphic classification of the spaces $\mathcal{K}\left(X, c_{0}(\Gamma)^{\eta}\right)$, where $\eta \geq \omega$ and $X$ belongs to a convenient class of Banach spaces.

As we will see, the study of this special case provides an extension of the classical isomorphic classification of the spaces $\mathbb{R}^{\eta}, \eta \geq \omega$ (Remark 1.2), as well a complete isomorphic classification of spaces $\mathcal{K}\left(\mathbb{R}^{\xi}, \mathbb{R}^{\eta}\right)$ with $\omega \leq \xi<\omega_{1}$ and $\eta \geq \omega$, which of course extends the above-mentioned result of C. Samuel (Remark 1.3), and also a complete classification, up to an isomorphism, of some spaces $\mathcal{K}\left(X, Y^{\eta}\right)$, where $\eta \geq \omega$ and $X$ and $Y$ are some of the spaces $l_{p}(\Gamma), 1 \leq p<\infty$, or $c_{0}(\Gamma)$ for some set $\Gamma$ (Remark 4.1).

We now turn our attention to the spaces $\mathcal{K}\left(X, c_{0}(\Gamma)^{\eta}\right), \eta \geq \omega$. Since $c_{0}(\Gamma)^{\eta}$ has the approximation property [3, Corollary 5, page 242] and [3, Example 11, page 245], it is well known [3], 7] that $\mathcal{K}\left(X, c_{0}(\Gamma)^{\eta}\right)$ is isomorphic to the injective tensor product $X^{*} \hat{\hat{\otimes}} c_{0}(\Gamma)^{\eta}$, where $X^{*}$ is the dual space of $X$. Moreover, by [3, Example 6, page 224] this last space is isomorphic to $c_{0}\left(\Gamma, X^{*}\right)^{\eta}$. One of the hypotheses needed on $X^{*}$ in order to obtain the isomorphic classification of the spaces $c_{0}\left(\Gamma, X^{*}\right)^{\eta}$ is that it has the Mazur property.

A Banach space $X$ is said to have the Mazur Property (in short, MP) if every element of $X^{* *}$, the bidual space of $X$, which is sequentially weak* continuous, is weak* continuous and thus is an element of $X$. Such spaces were investigated in [4, [12 and also in 9] and [19] where they were called $d$-complete and $\mu B$-spaces respectively. The class of Banach spaces having MP property includes the weakly compactly generated (WCG) spaces and therefore the spaces $l_{p}(\Gamma), 1 \leq p<\infty$, and $c_{o}(\Gamma)$ for some set $\Gamma$ [4, page 564].

In order to formulate our main result, we recall that the density character dens $X$ of a Banach space $X$ is the smallest cardinal number $\delta$ such that there exists a set of cardinality $\delta$ dense in $X$. The cardinality of an ordinal $\xi$ will be denoted by $\bar{\xi}$. The cardinality of a set $\Gamma$ will be denoted by $|\Gamma|$. We write $X \sim Y$ when the Banach spaces $X$ and $Y$ are isomorphic, and $X \not \psi Y$ otherwise. Thus, the aim of this paper is to prove the following result about the isomorphic classification of spaces $c_{0}(\Gamma, X)^{\eta}, \eta \geq \omega$.

Theorem 1.1. Let $X$ be a Banach space which contains no copy of $c_{0}, \Gamma$ a set, $\alpha$ an initial ordinal and $\xi \leq \eta$ two infinite ordinals. Then:

(1) If $c_{0}(\Gamma, X)^{\xi} \sim c_{0}(\Gamma, X)^{\eta}$ with $\bar{\eta}=\aleph_{1}$ and $X$ has $M P$, or $\bar{\eta}>\aleph_{1}$ and $|\Gamma|<\bar{\eta}$, then $\bar{\xi}=\bar{\eta}$.

(2) Suppose $\bar{\xi}=\bar{\eta}=\bar{\alpha}$ and assume that $\alpha=\omega$, or $\alpha$ is a singular ordinal with $|\Gamma|<\bar{\alpha}$, or $\alpha=\omega_{1},|\Gamma| \leq \aleph_{0}, \omega_{1}^{2} \leq \xi$ and $X$ has $M P$, or $\alpha$ is a regular ordinal with $\alpha>\omega_{1},|\bar{\Gamma}|$ dens $X^{*}<\bar{\alpha}, \alpha^{2} \leq \xi$ and $X$ has $M P$. Then $c_{0}(\Gamma, X)^{\xi} \sim c_{0}(\Gamma, X)^{\eta}$ if and only if $\eta<\xi^{\omega}$.

(3) Suppose that $\alpha$ is a nondenumerable regular ordinal, $\xi, \eta \in\left[\alpha, \alpha^{2}\right]$ and let $\xi^{\prime}, \eta^{\prime}, \gamma, \delta$ be ordinals such that $\xi=\alpha \xi^{\prime}+\gamma, \eta=\alpha \eta^{\prime}+\delta, \xi^{\prime}, \eta^{\prime} \leq \alpha$, $\gamma, \delta<\alpha$. Assume that $X$ has MP. If $\alpha=\omega_{1}$, or $\alpha>\omega_{1}$ and $|\Gamma|$ dens $X^{*}<\bar{\alpha}$, then $c_{0}(\Gamma, X)^{\xi} \sim c_{0}(\Gamma, X)^{\eta}$ if and only if $|\Gamma| \bar{\xi}^{\prime}<\aleph_{0}$ and $X^{|\Gamma| \bar{\xi}^{\prime}} \sim$ $X^{|\Gamma| \bar{\eta}^{\prime}}$ or $\aleph_{0} \leq|\Gamma| \bar{\xi}^{\prime}=|\Gamma| \bar{\eta}^{\prime}$. 
(4) Suppose that $\alpha$ is a nondenumerable regular ordinal, $\alpha \leq \xi<\alpha^{2} \leq \eta$ and assume that $X$ has $M P$. If $\alpha=\omega_{1}$ and $|\Gamma| \leq \aleph_{0}$, or $\alpha>\omega_{1}$ and $|\Gamma|$ dens $X^{*}<\bar{\alpha}$, then $c_{0}(\Gamma, X)^{\xi} \nsim c_{0}(\Gamma, X)^{\eta}$.

Remark 1.2. Notice that Theorem 1.1 provides a complete isomorphic classification of spaces $c_{0}(\Gamma, X)^{\eta}, \eta \geq \omega,|\Gamma| \leq \aleph_{0}$, whenever $X$ contains no copy of $c_{0}, X$ has MP and dens $X^{*} \leq \aleph_{1}$. In the case where $X=\mathbb{R}$ and $\Gamma$ has only one point, this result is due to Bessaga and Pełczyński [1], in the case where $\omega \leq \eta<\omega_{1}$; Semadeni [18, in the case where $\omega_{1} \leq \eta \leq \omega_{1} \omega$; and independently Kislyakov [10] and Gul'ko and Os'kin [8] in the general case.

Remark 1.3. We recall that if $X$ has MP, then $l_{1}(\Gamma, X)$ has MP whenever $|\Gamma|<m_{r}$, where $m_{r}$ denotes the least real-valued measurable cardinal [4, Theorem 5.10]. Moreover, we can prove by a standard gliding hump argument that if $X$ does not contain a copy of $c_{0}$, then $l_{1}(\Gamma, X)$ also does not contain a copy of $c_{0}$; see 2. Therefore, under the Continuous Hypothesis, $2^{\aleph_{0}}=\aleph_{1}$, and by using the well known $c_{0}-l_{1}-l_{\infty}$ Theorem of Bessaga and Pełczyński [11, Proposition 2.e.8], we see that Theorem 1.1 also gives a complete isomorphic classification of spaces $\mathcal{K}\left(X^{\xi}, c_{0}(\Gamma)^{\eta}\right) \sim c_{0}\left(\Gamma, l_{1}\left(\mathbb{N}, X^{*}\right)\right)^{\eta}, \omega \leq \xi<\omega_{1}, \eta \geq \omega$, where $|\Gamma| \leq \aleph_{0}, \mathbb{N}$ is the set of positive integers, $X$ contains no complemented copy of $l_{1}, X^{*}$ has MP and dens $X^{* *} \leq \aleph_{1}$. Observe that the Continuous Hypothesis is not necessary for the case where $X=\mathbb{R}, \Gamma$ has only one point and $\omega \leq \xi=\eta<\omega_{1}$. This is exactly the result of C. Samuel [17, which motivated this work.

\section{Some PRELIMINARY RESUlts}

The proof of Theorem 1.1 will be given as a consequence of some lemmas. Let $X$ and $Y$ be Banach spaces. By $X \hookrightarrow Y$ we mean that $Y$ contains a subspace isomorphic to $X$. First of all, notice that proceeding as in the proof of [17, Lemma 3.1] we get the following result.

Lemma 2.1. Let $X$ be a Banach space and $\eta$ an infinite ordinal. If $\mathbb{R}^{\eta^{\omega}} \hookrightarrow X^{\eta}$, then $\mathbb{R}^{\eta} \hookrightarrow X^{\gamma}$ for some ordinal $\gamma<\eta$.

Lemma 2.2. Let $X$ be a Banach space and suppose that $\mathbb{R}^{\omega^{\omega}} \hookrightarrow c_{0}(\Gamma, X)$ for some set $\Gamma$. Then $c_{0} \hookrightarrow X$.

Proof. Since $\mathbb{R}^{\omega^{\omega}}$ is separable, it is easy to see that there exists $\Gamma_{1} \subset \Gamma$ with $\left|\Gamma_{1}\right|=$ $\aleph_{0}$ such that $\mathbb{R}^{\omega^{\omega}} \hookrightarrow c_{0}\left(\Gamma_{1}, X\right)$. Furthermore, by [1, Lemma 1.2], $c_{0}\left(\Gamma_{1}, X\right) \sim X^{\omega}$. Therefore by Lemma $2.1, \mathbb{R}^{\omega} \hookrightarrow X^{m}$ for some $1 \leq m<\omega$. So according to [15, Theorem 1] and once again [1, Lemma 1.2], $c_{0} \sim \mathbb{R}^{\omega} \hookrightarrow X$.

Lemma 2.3. Let $X$ be a Banach space and $\alpha$ an infinite initial ordinal. Suppose that $\mathbb{R}^{\alpha} \hookrightarrow c_{0}(\Gamma, X)^{\eta}$ for some set $\Gamma$ with $|\Gamma|<\bar{\alpha}$ and $\eta<\alpha$. Then $c_{0} \hookrightarrow X$.

Proof. Let $I$ be the set of isolated points of $[1, \alpha]$. Then $c_{0}(I) \hookrightarrow \mathbb{R}^{\alpha}$, and since $c_{o}(\Gamma, X)^{\eta} \sim c_{0}(\Gamma) \hat{\hat{\otimes}} \mathbb{R}^{\eta} \hat{\hat{\otimes}} X \sim C(\gamma \Gamma) \hat{\hat{\otimes}} \mathbb{R}^{\eta} \hat{\hat{\otimes}} X \sim C(\gamma \Gamma \times[1, \eta], X)$, where $\gamma \Gamma$ is the Alexandroff compactification of the discrete set $\Gamma$, there exists an isomorphism $T$ from $c_{0}(I)$ onto a subspace of $C(\gamma \Gamma \times[1, \eta], X)$. Let $\left.M \in\right] 0,+\infty$ [ be such that $M \leq\|T(x)\|, \forall x \in c_{0}(I),\|x\|=1$. Denote by $\left(e_{i}\right)_{i \in I}$ the unit-vectors basis of $c_{0}(I)$; that is, $e_{i}(j)=1$ if $i=j$ and $e_{i}(j)=0$ if $i \neq j$, for all $i, j \in I$. Let $K$ be the set of isolated points of $\gamma \Gamma \times[1, \eta]$. Thus, by hypothesis, $|K|<\bar{\alpha}$. For a fixed $k \in K$, we define $I_{k}=\left\{i \in I: M / 2 \leq\left\|T\left(e_{i}\right)(k)\right\|\right\}$. Therefore $I=\bigcup_{k \in K} I_{k}$. 
Hence there is a $k \in K$ satisfying $\left|I_{k}\right| \geq \aleph_{0}$. We identify $c_{0}\left(I_{k}\right)$ with the subspace of $c_{0}(I)$ consisting of those elements $f$ such that $f(\gamma)=0$ for every $\gamma \notin I_{k}$. Let $P_{k}: C(\gamma \Gamma \times[1, \eta], X) \rightarrow X$ be the natural projection; that is, $P_{k}(f)=f(k)$, for all $f \in C(\gamma \Gamma \times[1, \eta], X)$. Next, consider the operator $L=P_{k} T_{\mid c_{0}\left(I_{k}\right)}: c_{0}\left(I_{k}\right) \rightarrow X$. Then $\inf \left\{\left\|L\left(e_{i}\right)\right\|: i \in I_{k}\right\}>0$. So, according to Remark 1 which follows [13, Theorem 3.4], there exists $J \subset I_{k}$ with $|J|=\left|I_{k}\right|$ such that $L_{\mid c_{0}(J)}$ is an isomorphism onto its image.

Analogously, we have

Lemma 2.4. Let $X$ be a Banach space such that $c_{0}(I) \hookrightarrow c_{0}(J, X)$ for some sets $I$ and $J$ with $|J|<|I|$ and $|I| \geq \aleph_{0}$. Then $c_{0} \hookrightarrow X$.

To state the next lemma, we need to recall some Banach spaces introduced in [5]. Let $\gamma$ be an ordinal. $A \gamma$-sequence in a set $A$ is a function $f:[1, \gamma[\rightarrow A$ and will be denoted by $\left(x_{\theta}\right)_{\theta<\gamma}$. Let $\alpha$ be a regular ordinal, $\alpha \geq \omega_{1}, \varphi$ any ordinal and $X$ a Banach space. By $X_{\alpha}^{\varphi}$ we will denote the space of all $x^{* *} \in X^{* *}$ having the following property: for every limit ordinal $\beta<\alpha$ and for every $\varphi$-sequence $x^{\eta}=\left(x_{\xi}^{*}(\eta)\right)_{\xi<\beta}$ of $\beta$-sequences of $X^{*}$ such that there exists $M \in \mathbb{R}$ with $\left\|x_{\xi}^{*}(\eta)\right\|$ $\leq M, \forall \eta<\varphi, \forall \xi<\beta$ and such that $x_{\xi}^{*}(\eta)(x) \stackrel{\xi \rightarrow \beta}{\longrightarrow} 0, \forall x \in X$, uniformly in $\eta$, we have $x^{* *}\left(x_{\xi}^{*}(\eta)\right) \stackrel{\xi \rightarrow \beta}{\longrightarrow} 0$ uniformly in $\eta$. If $X$ is a Banach space, then $c X$ denotes the canonical image of $X$ in $X^{* *}$.

By using the Hahn-Banach theorem as in the proof of [10, Lemma 1.4] it is straightforward to prove:

Lemma 2.5. Let $X$ be a closed subspace of the Banach space $Y, \alpha$ a regular ordinal and $\varphi$ any ordinal. Then $\frac{X_{\alpha}^{\varphi}}{c X} \hookrightarrow \frac{Y_{\alpha}^{\varphi}}{c Y}$.

Lemma 2.6. Let $X$ be a Banach space having $M P, \alpha$ a nondenumerable regular ordinal and $\Gamma$ a set. Suppose that $\alpha=\omega_{1}$ and $|\Gamma| \leq \aleph_{0}$, or $\alpha>\omega_{1}$ and $|\Gamma|$ dens $X^{*}<\bar{\alpha}$. If $\mathbb{R}^{\alpha^{2}} \hookrightarrow c_{0}(\Gamma, X)^{\eta}$ for some $\eta<\alpha^{2}$, then $c_{0} \hookrightarrow X$.

Proof. We will distinguish two cases.

Case 1. $\eta<\alpha$. In this case, it follows from $\mathbb{R}^{\alpha} \hookrightarrow c_{0}(\Gamma, X)^{\eta}$ and Lemma 2.3 that $c_{0} \hookrightarrow X$.

Case 2. $\alpha \leq \eta<\alpha^{2}$. Thus $\eta=\alpha \xi+\theta$ for some ordinals $\xi<\alpha$ and $\theta<\alpha$. Since that $\mathbb{R}^{\eta} \sim \mathbb{R}^{\alpha \xi}$ [10, Theorem 3.1], we have

$$
\mathbb{R}^{\alpha^{2}} \hookrightarrow c_{0}(\Gamma, X)^{\eta} \sim c_{0}(\Gamma) \hat{\hat{\otimes}} \mathbb{R}^{\eta \hat{\hat{\otimes}}} X \sim c_{0}(\Gamma) \hat{\hat{\otimes}} \mathbb{R}^{\alpha \xi} \hat{\hat{\otimes}} X \sim c_{0}(\Gamma, X)^{\alpha \xi} .
$$

Pick $\varphi$ the ordinal defined by

$$
\varphi=\left\{\begin{array}{l}
\omega \text { if } \alpha=\omega_{1}, \\
\text { the first ordinal of cardinality }|\Gamma| \text { dens } X^{*} \text { if } \alpha>\omega_{1} .
\end{array}\right.
$$

Let $I$ and $J$ be two sets with $|I|=\bar{\alpha}$ and $|J|=\bar{\xi}$. According to Lemma 2.5, [5, Corollary 2.8], [5. Remark 2.7] and [6, Lemma 3.2], we infer that

$$
c_{0}(I) \sim \frac{\left(\mathbb{R}^{\alpha^{2}}\right)_{\alpha}^{\varphi}}{c \mathbb{R}^{\alpha^{2}}} \hookrightarrow \frac{\left(c_{0}(\Gamma, X)^{\alpha \xi}\right)_{\alpha}^{\varphi}}{c c_{0}(\Gamma, X)^{\alpha \xi}} \sim c_{0}\left(J, c_{0}(\Gamma, X)\right) .
$$

Thus it is enough to use Lemma 2.4 to deduce $c_{0} \hookrightarrow X$. 
The next result is an extension of [17, Theorem 3.2].

Lemma 2.7. Let $\alpha$ be an initial ordinal and $\xi \leq \eta$ ordinals with $\bar{\xi}=\bar{\eta}=\bar{\alpha}$. Put $\alpha_{0}=\omega^{\omega}$ if $\alpha=\omega, \alpha_{0}=\alpha$ if $\alpha$ is a singular ordinal and $\alpha_{0}=\alpha^{2}$ if $\alpha$ is a regular ordinal, $\alpha \geq \omega_{1}$. Suppose that $X$ is a Banach space having MP and containing no copy of $c_{0}$ and $\Gamma$ is a set satisfying

(1) $\Gamma$ is arbitrary if $\alpha=\omega$,

(2) $|\Gamma|<\bar{\alpha}$ if $\alpha$ is a singular ordinal,

(3) $|\Gamma| \leq \aleph_{0}$ if $\alpha=\omega_{1}$,

(4) $|\Gamma|$ dens $X^{*}<\bar{\alpha}$ if $\alpha$ is a regular ordinal with $\alpha>\omega_{1}$.

If $\mathbb{R}^{\eta} \hookrightarrow c_{0}(\Gamma, X)^{\xi}$ with $\alpha_{0} \leq \xi$, then $\mathbb{R}^{\eta} \hookrightarrow \mathbb{R}^{\xi}$.

Proof. We introduce two sets of ordinals

$$
\begin{aligned}
& I_{1}=\left\{\theta: \bar{\theta}=\bar{\alpha}, \alpha_{0} \leq \theta, \mathbb{R}^{\theta} \nrightarrow \mathbb{R}^{\gamma}, \forall \gamma<\theta\right\}, \\
& I_{2}=\left\{\theta: \bar{\theta}=\bar{\alpha}, \alpha_{0} \leq \theta, \mathbb{R}^{\theta} \nrightarrow c_{0}(\Gamma, X)^{\gamma}, \forall \gamma<\theta\right\} .
\end{aligned}
$$

Initially we will prove that $I_{1}=I_{2}$. Clearly $I_{2} \subset I_{1}$. Observe that by Lemmas 2.2, 2.3 and 2.6, we deduce that $\alpha_{0} \in I_{2}$. Now, assume that $I_{2}$ is a proper subset of $I_{1}$. Let $\alpha_{1}$ be the least element of $I_{1} \backslash I_{2}$. We have $\alpha_{0}<\alpha_{1}$. Since $\alpha_{1} \notin I_{2}$, there exists an ordinal $\gamma_{1}<\alpha_{1}$ such that $\mathbb{R}^{\alpha_{1}} \hookrightarrow c_{0}(\Gamma, X)^{\gamma_{1}}$.

Let $\alpha_{2}=\min \left\{\gamma, \alpha_{0} \leq \gamma<\alpha_{1}: \mathbb{R}^{\alpha_{1}} \hookrightarrow c_{0}(\Gamma, X)^{\gamma}\right\}$. We have $\alpha_{2} \leq \gamma_{1}$. Now, we will show that $\alpha_{2} \in I_{1}$. If this is not the case, there exists an ordinal $\gamma_{2}<\alpha_{2}$ such that $\mathbb{R}^{\alpha_{2}} \hookrightarrow \mathbb{R}^{\gamma_{2}}$. Therefore

$$
c_{0}(\Gamma, X)^{\alpha_{2}} \sim c_{0}(\Gamma) \hat{\hat{\otimes}} \mathbb{R}^{\alpha_{2}} \hat{\hat{\otimes}} X \hookrightarrow c_{0}(\Gamma) \hat{\hat{\otimes}} \mathbb{R}^{\gamma_{2}} \hat{\hat{\otimes}} X \sim c_{0}(\Gamma, X)^{\gamma_{2}} .
$$

Consequently $\mathbb{R}^{\alpha_{1}} \hookrightarrow c_{0}(\Gamma, X)^{\gamma_{2}}$, in contradiction with the definition of $\alpha_{2}$.

So $\alpha_{2} \in I_{1}$ and, since $\alpha_{2}<\alpha_{1}$, it follows from the definition of $\alpha_{1}$ that $\alpha_{2} \in I_{2}$. That is, $\mathbb{R}^{\alpha_{2}} \& c_{0}(\Gamma, X)^{\gamma}, \forall \gamma<\alpha_{2}$. Thus by Lemma 2.1, we conclude that $\mathbb{R}^{\alpha_{2}^{\omega}} \hookrightarrow c_{0}(\Gamma, X)^{\alpha_{2}}$.

On the other hand, note that if $\alpha_{1}<\alpha_{2}{ }^{\omega}$, then by [10, Theorem 2.1], $\mathbb{R}^{\alpha_{1}} \sim \mathbb{R}^{\alpha_{2}}$, which is absurd by the definition of $\alpha_{1}$. Consequently $\alpha_{2}{ }^{\omega} \leq \alpha_{1}$ and $\mathbb{R}^{\alpha_{2}^{\omega}} \hookrightarrow$ $\mathbb{R}^{\alpha_{1}}$. Furthermore, by the definition of $\alpha_{2}, \mathbb{R}^{\alpha_{1}} \hookrightarrow c_{0}(\Gamma, X)^{\alpha_{2}}$. Therefore $\mathbb{R}^{\alpha_{2}^{\omega}} \hookrightarrow$ $c_{0}(\Gamma, X)^{\alpha_{2}}$, in contradiction with what we have just proved above. Hence $I_{1}=I_{2}$.

Next, to complete the proof of the lemma, suppose that $\mathbb{R}^{\eta} \leftrightarrow \mathbb{R}^{\xi}$ and let $\xi_{1}=\min \left\{\theta: \mathbb{R}^{\eta} \hookrightarrow \mathbb{R}^{\theta}\right\}$. Hence $\xi<\xi_{1} \leq \eta$ and $\mathbb{R}^{\xi_{1}} \hookrightarrow \mathbb{R}^{\gamma}, \forall \gamma<\xi_{1}$. In particular, $\xi_{1} \in I_{1}=I_{2}$, which is absurd, because $\mathbb{R}^{\xi_{1}} \hookrightarrow \mathbb{R}^{\eta} \hookrightarrow c_{0}(\Gamma, X)^{\xi}$.

\section{Proof of Theorem 1.1}

(1) First suppose that $\bar{\eta}=\aleph_{1}$ and $X$ has MP. Assume that $\bar{\xi}=\aleph_{0}$. Then $\mathbb{R}^{\omega_{1}} \hookrightarrow$ $\mathbb{R}^{\eta} \hookrightarrow c_{0}(\Gamma, X)^{\xi}$, which is absurd, because $c_{0}(\Gamma, X)^{\xi}$ has MP [9, Corollary 5.2] and $\mathbb{R}^{\omega_{1}}$ does not have MP [19, page 49].

Now suppose that $\bar{\eta}>\aleph_{1}$ and $|\Gamma|<\bar{\eta}$. Assume that $\bar{\xi}<\bar{\eta}$ and let $\alpha$ be the initial ordinal of cardinality $\bar{\eta}$. Then $\mathbb{R}^{\alpha} \hookrightarrow \mathbb{R}^{\eta} \hookrightarrow c_{0}(\Gamma, X)^{\xi}$ and by Lemma 2.3, $c_{0} \hookrightarrow X$, but this is a contradiction.

To prove the sufficiency of statements (2) and (3) it suffices to keep in mind [10, Theorem 1] and [10, Theorem 2] and observe that if $\mathbb{R}^{\xi} \sim \mathbb{R}^{\eta}$, then

$c_{0}(\Gamma, X)^{\xi} \sim c_{0}(\Gamma) \hat{\hat{\otimes}} X^{\xi} \sim c_{0}(\Gamma) \hat{\hat{\otimes}} X \hat{\hat{\otimes}} \mathbb{R}^{\xi} \sim c_{0}(\Gamma) \hat{\hat{\otimes}} X \hat{\hat{\otimes}} \mathbb{R}^{\eta} \sim c_{0}(\Gamma) \hat{\hat{\otimes}} X^{\eta} \sim c_{0}(\Gamma, X)^{\eta}$. Next we prove the necessity of statements (2) and (3). 
(2) First assume that $C(\gamma \Gamma, X)^{\eta} \sim C(\gamma \Gamma, X)^{\xi}$. We distinguish two cases:

Case 1. $\alpha=\omega$. If $\xi \geq \omega^{\omega}$, then by hypothesis $\mathbb{R}^{\eta} \hookrightarrow c_{0}(\Gamma, X)^{\xi}$. Thus Lemma 2.7 implies that $\mathbb{R}^{\eta} \hookrightarrow \mathbb{R}^{\xi}$, and by [10, Theorem 1] we have $\eta<\xi^{\omega}$. On the other hand, if $\xi<\omega^{\omega}$, again by [10, Theorem 1], we conclude that $\mathbb{R}^{\xi} \sim \mathbb{R}^{\omega}$. Let $\Gamma_{1}$ be a set of cardinality $|\Gamma| \aleph_{0}$. Then $c_{0}\left(\Gamma_{1}, X\right) \sim c_{0}(\Gamma, X)^{\omega} \sim c_{0}\left(\Gamma_{1}, X\right)^{\xi} \sim$ $c_{0}\left(\Gamma_{1}, X\right)^{\eta}$. Therefore, if $\eta>\xi^{\omega} \geq \omega^{\omega}$, we would have $\mathbb{R}^{\omega} \hookrightarrow \mathbb{R}^{\eta} \hookrightarrow c_{0}\left(\Gamma_{1}, X\right)$. Thus Lemma 2.2 would imply that $c_{0} \hookrightarrow X$, which is absurd.

Case 2. $\alpha>\omega$. If $\eta>\xi^{\omega}$, then $\mathbb{R}^{\eta} \hookrightarrow c_{0}(\Gamma, X)^{\eta} \sim c_{0}(\Gamma, X)^{\xi}$. Since the hypotheses of Lemma 2.7 hold, we deduce $\mathbb{R}^{\eta} \hookrightarrow \mathbb{R}^{\xi}$, which is absurd by [10, Theorem 1].

(3) Let $I$ and $J$ be two sets with $|I|=\bar{\xi}^{\prime}$ and $|J|=\bar{\eta}^{\prime}$. Let $\varphi$ be as in the proof of Lemma 2.6. By [5, Remark 2.3], [5, Corollary 2.8], [5, Remark 2.7] and 6, Lemma 3.2],

$$
c_{0}\left(I, c_{0}(\Gamma, X)\right) \sim \frac{\left(c_{0}(\Gamma, X)^{\alpha \xi^{\prime}}\right)_{\alpha}^{\varphi}}{c c_{0}(\Gamma, X)^{\alpha \xi^{\prime}}} \sim \frac{\left(c_{0}(\Gamma, X)^{\alpha \eta^{\prime}}\right)_{\alpha}^{\varphi}}{c c_{0}(\Gamma, X)^{\alpha \eta^{\prime}}} \sim c_{0}\left(J, c_{0}(\Gamma, X)\right) .
$$

Hence if $|\Gamma| \bar{\xi}^{\prime}$ and $|\Gamma| \bar{\eta}^{\prime}$ are finite, then $X^{|\Gamma| \bar{\xi}^{\prime}} \sim X^{|\Gamma|} \bar{\eta}^{\prime}$. On the other hand, if $|\Gamma| \bar{\xi}^{\prime}$ or $|\Gamma| \bar{\eta}^{\prime}$ is infinite, then applying Lemma 2.4 we see that $|\Gamma| \bar{\xi}^{\prime}=|\Gamma| \bar{\eta}^{\prime}$.

(4) Assume that $c_{0}(\Gamma, X)^{\xi} \sim c_{0}(\Gamma, X)^{\eta}$ with $\alpha \leq \xi<\alpha^{2} \leq \eta$. Then $\mathbb{R}^{\alpha^{2}} \hookrightarrow$ $c_{0}(\Gamma, X)^{\xi}$. Hence by Lemma 2.6, $c_{0} \hookrightarrow X$.

This completes the proof of Theorem 1.1.

\section{Final REMARKS AND SOME PROBLEMS}

Remark 4.1. Let $1<p<\infty, 1<q<\infty$ and $I$ and $J$ be two sets. Recall that the spaces $l_{p}(I), c_{0}(I)$ and $l_{1}(I)$ have the approximation property [3, Example 11, page 245]. Moreover, the spaces $l_{1}(I) \hat{\hat{\otimes}} l_{1}(J), l_{p}(I) \hat{\hat{\otimes}} l_{1}(J)$ and $l_{p}(I) \hat{\hat{\otimes}} l_{q}(J)$ with $p^{\prime}>q$, where $1 / p+1 / p^{\prime}=1$, do not contain a copy of $c_{0}$ [16. Main Theorem].

Finally, the spaces $l_{p}(I) \hat{\hat{\otimes}} l_{q}(J), l_{p}(I) \hat{\hat{\otimes}} l_{1}$ and $l_{1} \hat{\hat{\otimes}} l_{1}$ have MP [9, Corollary 5.2.1] and 9, Corollary 5.2.3]. Hence Remarks 1.2 and 1.3 provide a complete isomorphic classification of the following spaces:

(1) $\mathcal{K}\left(l_{p}(I), c_{0}(J)^{\eta}\right)$, where $\eta \geq \omega,|I| \leq \aleph_{1}$ and $|J| \leq \aleph_{0}$.

(2) $\mathcal{K}\left(c_{0}(I), c_{0}(J)^{\eta}\right)$, where $\eta \geq \omega,|I| \leq \aleph_{0}$ and $|J| \leq \aleph_{0}$.

(3) $\mathcal{K}\left(l_{p}(I), l_{q}(J)^{\eta}\right)$, where $\eta \geq \omega,|I| \leq \aleph_{1},|J| \leq \aleph_{1}$ and $p>q$.

(4) $\mathcal{K}\left(c_{0}(I), l_{p}(J)^{\eta}\right)$, where $\eta \geq \omega,|I| \leq \aleph_{0}$ and $|J| \leq \aleph_{1}$.

(5) $\mathcal{K}\left(l_{p}(I), l_{1}(J)^{\eta}\right)$, where $\eta \geq \omega,|I| \leq \aleph_{1}$ and $|J| \leq \aleph_{0}$.

(6) $\mathcal{K}\left(c_{0}(I), l_{1}(J)^{\eta}\right)$, where $\eta \geq \omega,|I| \leq \aleph_{0}$ and $|J| \leq \aleph_{0}$.

Remark 4.1 leads naturally to the following problems:

Problem 4.2. Classify, up to an isomorphism, the following spaces:

(1) $\mathcal{K}\left(l_{1}, c_{0}^{\eta}\right)$, where $\eta \geq \omega$.

(2) $\mathcal{K}\left(l_{1}, l_{p}^{\eta}\right)$, where $\eta \geq \omega$ and $1 \leq p<\infty$.

(3) $\mathcal{K}\left(l_{p}, l_{q}^{\eta}\right)$, where $\eta \geq \omega$ and $1<p \leq q<\infty$.

Finally, denote by $\beta \mathbb{N}$ the Stone-Cech compactification of $\mathbb{N}$. Since there exists a continuous function of $\beta \mathbb{N}$ onto $\beta \mathbb{N} \times \beta \mathbb{N}$, it follows that $C(\beta \mathbb{N}) \hat{\hat{\otimes}} C(\beta \mathbb{N}) \sim$ $C(\beta \mathbb{N} \times \beta \mathbb{N}) \hookrightarrow C(\beta \mathbb{N})$, and therefore $c_{0}(\mathbb{N}, C(\beta \mathbb{N})) \hat{\hat{\otimes}} c_{0}(\mathbb{N}, C(\beta \mathbb{N})) \hookrightarrow c_{0}(\mathbb{N}, C(\beta \mathbb{N}))$. Furthermore $\mathbb{R}^{\alpha} \hookrightarrow C(\beta \mathbb{N})$, for every $\alpha \geq \omega_{1}$; otherwise $c_{0}(\Gamma) \hookrightarrow C(\beta \mathbb{N})$, for some 
set $\Gamma$ with $|\Gamma|=\aleph_{1}$, which is absurd because $\beta \mathbb{N}$ satisfies the Countable Chain Condition [14, page 229]. Consequently the next proposition provides a partial solution to Problem 4.2(1).

Proposition 4.3. Let $\alpha$ be an infinite initial ordinal and $\xi \leq \eta$ with $\bar{\xi}=\bar{\eta}=\bar{\alpha}$. If $X$ is a Banach space satisfying $X \hat{\hat{\otimes}} X \hookrightarrow X, \mathbb{R}^{\alpha} \hookrightarrow X^{m}$ for every $1 \leq m<\omega$ and $X^{\xi} \sim X^{\eta}$, then $\eta<\xi^{\omega}$.

Proof. Suppose that $\eta \geq \xi^{\omega}$. Then $\mathbb{R}^{\xi^{\omega}} \hookrightarrow X^{\xi}$. Let

$$
\xi_{1}=\min \left\{\gamma: \gamma \leq \xi, \mathbb{R}^{\xi^{\omega}} \hookrightarrow X^{\gamma}\right\} .
$$

By hypothesis $\xi_{1}$ must be infinite, and since $\xi_{1} \leq \xi$, we infer that $\mathbb{R}_{1}^{\omega} \hookrightarrow \mathbb{R}^{\xi^{\omega}} \hookrightarrow$ $X^{\xi_{1}}$. Hence according to Lemma 2.1, $\mathbb{R}^{\xi_{1}} \hookrightarrow X^{\gamma_{1}}$ for some $\gamma_{1}<\xi_{1}$. Thus

$$
X^{\xi_{1}} \sim \mathbb{R}^{\xi_{1}} \hat{\hat{\otimes}} X \hookrightarrow X^{\gamma_{1}} \hat{\hat{\otimes}} X=(X \hat{\hat{\otimes}} X)^{\gamma_{1}} \hookrightarrow X^{\gamma_{1}} .
$$

Consequently, $\mathbb{R}^{\xi_{1}^{\omega}} \hookrightarrow X^{\xi_{1}} \hookrightarrow X^{\gamma_{1}}$, which is absurd by the definition of $\xi_{1}$.

\section{ACKNOWLEDGMENT}

The author would like to thank the referee for very helpful comments.

\section{REFERENCES}

1. C. Bessaga, A. Pełczyński, Spaces of continuous functions. IV, Studia Math. XIX (1960), 53-62. MR0113132 (22:3971)

2. L. Burlando, On subspaces of direct sums of infinite sequences of Banach spaces. Atti Accad. Ligure Sci. Lett. 46 (1989), 96-105. MR.1098789 (92b:46019)

3. J. Diestel, J.J. Uhl, Jr., Vector Measures, Mathematical Surveys 15, Amer. Math. Soc., Providence, RI (1977). MR0453964 (56:12216)

4. G. A. Edgar, Measurability in a Banach space. II, Indiana Univ. Math. J. 28 (1979), 559-579. MR542944 (81d:28016)

5. E. M. Galego, How to generate new Banach spaces non-isomorphic to their Cartesian squares, Bull. Polish Acad. Sci. Math. 47 (1999), 1, 21-25. MR1685684 (2001b:46015)

6. E. M. Galego, Banach spaces of continuous vector-valued functions of ordinals, Proc. Edinb. Math. Soc. (2) 44 (2001), 1, 49-62. MR.1879208 (2002k:46064)

7. A. Grothendieck, Produits tensoriels topologiques et espaces nucléaires, Mem. Amer. Math. Soc. 1955 (1955), no. 16. MR.0075539(17:763c)

8. S. P. Gul'ko, A. V. Os'kin, Isomorphic classification of spaces of continuous functions on totally ordered bicompacta, Functional Anal. Appl. 9 (1975), 56-57. MR0377489 (51:13661)

9. T. Kappeler, Banach spaces with the condition of Mazur, Math. Z. 191 (1986), 623-631. MR832820 (87h:46040)

10. S. V. Kislyakov, Classification of spaces of continuous functions of ordinals, Siberian Math. J. 16 (1975), no. 2, 226-231.

11. J. Lindenstrauss, L. Tzafriri, Classical Banach spaces. I. Sequence Spaces, Springer-Verlag, Berlin-New York (1977). MR0500056 (58:17766)

12. D. Leung, On Banach spaces with Mazur's property, Glasgow Math. J. 33 (1991), 51-54. MR 1089953 (92b:46021)

13. H. P. Rosenthal, On relatively disjoint families of measures, with some applications to Banach space theory, Studia Math. 37 (1970), 13-36. MR0270122(42:5015)

14. H. P. Rosenthal, On injective Banach spaces and the spaces $L^{\infty}(\mu)$ for finite measures $\mu$, Acta Math. 124 (1970), 205-248. MR0257721 (41:2370)

15. C. Samuel, Sur la reproductibilité des espaces $l_{p}$, Math. Scand. 45 (1979), 103-117. MR567436 (81e:46062)

16. C. Samuel, Sur les sous-espaces de $l_{p} \hat{\hat{\otimes}} l_{q}$. Math. Scand. 47 (1980), 247-250. MR612698 (82i:46029)

17. C. Samuel, On spaces of operators on $C(Q)$ spaces ( $Q$ countable metric space), Proc. Amer. Math. Soc. 137 (2009), no. 3, 965-970. MR2457436 
18. Z. Semadeni, Banach spaces non-isomorphic to their Cartesian squares. II, Bull. Acad. Polon. Sci. Sér. Sci. Math. Astr. Phys. 8 (1960), 81-84. MR0115074(22:5877)

19. A. Wilansky, Mazur spaces, Internat. J. Math. Math. Sci. 4 (1981), 39-53. MR606656 (82f:46001)

Department of Mathematics, University of São Paulo, São Paulo, Brazil 05508-090

E-mail address: eloi@ime.usp.br 